\title{
Iatrogenic pancreatitis in familial adenomatous polyposis
}

\author{
K P Nugent, A D Spigelman, C B Williams, R K S Phillips
}

\author{
Abstract \\ The first case of a patient with familial \\ adenomatous polyposis (FAP) who developed \\ pancreatitis after routine screening and biopsy \\ of the ampulla of Vater is described. \\ (Gut 1993; 34: 1269-1270)
}

Upper gastrointestinal tract malignancy is a major cause of death in patients with familial adenomatous polyposis (FAP). ${ }^{12}$ Regular screening is therefore advocated to determine those at greatest risk and to start treatment early; as well as to study the natural history of duodenal adenomas. ${ }^{3}$ Representative biopsies are taken of abnormal areas with histological examination to discover the degree of dysplasia and type of adenoma (tubular, tubulovillous, or villous). These two factors, as well as the size and number of polyps, are, as they are in the large bowel, thought to be associated with a greater risk of malignant change. ${ }^{4}$ The papillary area is commonly affected and is therefore the area where biopsies are usually carried out.

We report the first case of a patient with FAP who developed pancreatitis after routine screening and biopsy of the ampulla of Vater.

\section{Case report}

A 71 year old asymptomatic woman attended for a routine day case screening duodenoscopy using a side viewing video endoscope (Olympus EVIS JFV10). She had had a colectomy and ileorectal anastomosis for FAP in 1961 and had required 20 fulgurations for rectal polyps. She was not taking any drugs. Her first upper gastrointestinal endoscopy in 1988 had shown more than 20 polyps, between $0.5 \mathrm{~mm}$ and $5 \mathrm{~mm}$ in size. Histological examination at this time showed the papilla to have mild dysplasia and duodenal polyps to be tubular adenomas with mild dysplasia. Biopsy specimens were taken with $7 \mathrm{~mm}$ forceps (Olympus FB-24U).

She returned for her follow up endoscopy in 1992 and once again had more than 20 polyps, the largest being $5 \mathrm{~mm}$ in size. Biopsy specimens were taken of suspicious areas including a $5 \mathrm{~mm}$ polyp on the papilla (this was a tubular adenoma with mild dysplasia). Biopsy specimens were taken with new rat toothed flexible head biopsy forceps (Olympus FB-40Q). Within eight hours she had developed central abdominal pain, vomiting, and distension. She was seen to be afebrile with a tender rigid abdomen on admission to her local hospital. An erect abdominal $x$ ray showed no free gas but a presumptive diagnosis of perforation was made. Unfortunately plasma amylase activities were not assessed before or after surgery. At laparotomy, however, she was found to have an area of oedematous, necrotic, haemorrhagic pancreas adjacent to the second part of the duodenum. There was no perforation seen. She was treated with fluids and analgesia. She recovered well and was discharged eight days later.

\section{Discussion}

This is the first published case of iatrogenic pancreatitis in FAP. FAP patients are at $100-300$ times greater risk than the general population of dying from upper gastrointestinal malignancies. ${ }^{2}$ When treated with a prophylactic colectomy the colon cancer risk is eliminated and the rectal cancer risk is minimised by regular surveillance and fulguration of rectal polyps. Duodenal malignancy then becomes the greatest cause of death in patients with FAP's ; therefore identification of patients most at risk of malignant change of the duodenum is of great importance. At present the best way of monitoring these patients is by endoscopy. The incidence of pancreatitis after endoscopic retrograde cholangiopancreatographic and sphincterotomy requiring hospital admission is approximately $1 \%$; although the serum amylase activity is almost always raised. ${ }^{6}$ The incidence of pancreatitis after endoscopic biopsy of the papilla is unknown.

Even without endoscopic intervention, patients with FAP are at risk of developing acute pancreatitis. In a series of 141 patients who had had a prophylactic colectomy and ileorectal anastomosis, ${ }^{7}$ five patients had a history of acute pancreatitis. Histological information was available in two of these patients; both showed coexisting pancreatic abnormalities (an $8 \mathrm{~mm}$ adenoma in the mid section of the pancreas with a dilated duct and an adenocarcinoma at the ampulla of Vater). Indeed, adenomas and dysplastic areas may occur throughout the biliary and pancreatic tree in patients with $\mathrm{FAP}^{8}$ and these may predispose such patients to pancreatitis. ${ }^{9} 10$

Like all screening programmes endoscopic surveillance of the duodenum in FAP patients seeks to detect treatable lesions at an asymptomatic stage and this has been achieved. "Despite targeting this relatively small but high risk group, such screening programmes have been the subject of criticism. ${ }^{12}$ The anti-screening argument would be bolstered if the complication rate of examinations was high.

This is the first complication, however, that we have encountered in 150 patients in whom biopsies have been performed. Perhaps the rat toothed forceps used in the present case contributed to the onset of pancreatitis as they had not been used before. Numerous further endoscopic biopsies of enlarged papillas in FAP patients 
have since been performed with standard forceps without complication. As almost $90 \%$ of papillas in FAP patients show visible enlargement or deformity suggestive of dysplasia we consider it ethical and clinically desirable to continue such histological assessment.

1 Jagelman DG, DeCosse JJ, Bussey HJR. Upper gastrointestinal cancer in familial adenomatous polyposis. Lance 1988; i: 1149-5

2 Offerhaus GJA, Giardiello FM, Krush AJ, Booker SV, Tersmette AC, Kelley NC, et al. The risk of uppe gastrointestinal cancer in familial adenomatous polyposis Gastroenterology 1992; 102: 1980-2.

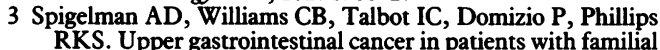
adenomatous polyposis. Lancet 1989; ii: 783-5.

4 Sellner $F$. Investigations on the significance of the adenoma-carcinoma sequence in the small bowel. Cancer 1990; 66: 702-15.
5 Arvanitis ML, Jagelman DG, Fazio VW, Lavery IC, McGannon E. Mortality in patients with familial adenomatous polyposis. Dis Colon Rectum 1990; 33: 639-42.

6 Hart R, Classen M. Complications of diagnostic gastrointestinal endoscopy. Endoscopy 1990; 22: 229-33.

7 Sener SF, Miller $\mathrm{HH}$. The spectrum of polyposis. Surg Gynecol Obstet 1984; 159: 525-32.

8 Spigelman AD, Farmer KCR, James M, Richman PI, Phillips RKS. Tumours of the liver, bile ducts, pancreas and RKS. Tumours of the liver, bile ducts, pancreas and duodenum in a single patient with fam
polyposis. Br $\mathcal{F}$ Surg 1991; 78: 979-80.

9 Berk T, Friedman LS, Goldstein SD, Marks GP, Rosato FE Relapsing acute pancreatitis as the presenting manifestation of an ampullary neoplasm in a patient with familial polyposis coli. Am ₹ Gastroentrol 1985; 80: 627-9.

10 Burt RW, Rikkers LF, Gardner EJ, Lee RG, Tolman KG Villous adenoma of the duodenal papilla presenting as necrotising pancreatitis in a patient with Gardner's syndrome. Gastroenterology 1987; 92: 532-5.

11 Spigelman AD, Jensen W, Bulow S. Duodenal cancer in polyposis patients. Gastroenterology 1992; 103: 1995.

12 Norfleet RG. Screening for upper gastrointestinal neoplasms orfleet RG. Screening for upper gastrointestinal neoplasm in patients with familial adenomatous polyposis and
Gardner's syndrome. $\mathcal{F}$ Clin Gastroenterology 1992; 14: 95-6. 